\title{
Coherent dynamics of interwell excitons in GaAs/AlxGa1-xAs superlattices
}

\author{
Mizeikis, V.; Birkedal, Dan; Langbein, Wolfgang Werner; Hvam, Jørn Märcher
}

Published in:

Physical Review B

Link to article, DOI:

10.1103/PhysRevB.55.7743

Publication date:

1997

Document Version

Publisher's PDF, also known as Version of record

Link back to DTU Orbit

Citation (APA):

Mizeikis, V., Birkedal, D., Langbein, W. W., \& Hvam, J. M. (1997). Coherent dynamics of interwell excitons in GaAs/AlxGa1-xAs superlattices. Physical Review B, 55(12), 7743-7748.

https://doi.org/10.1103/PhysRevB.55.7743

\section{General rights}

Copyright and moral rights for the publications made accessible in the public portal are retained by the authors and/or other copyright owners and it is a condition of accessing publications that users recognise and abide by the legal requirements associated with these rights.

- Users may download and print one copy of any publication from the public portal for the purpose of private study or research.

- You may not further distribute the material or use it for any profit-making activity or commercial gain

- You may freely distribute the URL identifying the publication in the public portal 


\title{
Coherent dynamics of interwell excitons in $\mathbf{G a A s} / \mathrm{Al}_{x} \mathbf{G a}_{1-x} \mathrm{As}$ superlattices
}

\author{
V. Mizeikis, D. Birkedal, W. Langbein, and J. M. Hvam \\ Mikroelektronik Centret, The Technical University of Denmark, DK-2800 Lyngby, Denmark
}

(Received 6 August 1996)

\begin{abstract}
Coherent exciton dynamics in a GaAs/ $\mathrm{Al}_{x} \mathrm{Ga}_{1-x} \mathrm{As}$ narrow-miniband superlattice is studied by spectrally resolved transient four-wave mixing. Coherent optical properties of the investigated structure are found to be strongly affected by the existence of two different heavy-hole excitonic states. One of them, the $1 s$ heavy-hole exciton, is almost identical to the same state in noninteracting quantum wells, while the other, the heavy-hole interwell exciton, is composed of an electron and a heavy hole in adjacent wells. The interwell exciton leads to a resonant enhancement in the four-wave mixing spectra and exhibits quantum beats with the $1 s$ heavy-hole exciton. The dephasing of the interwell exciton is one order of magnitude faster than that of the heavy-hole exciton and is mostly due to intensity-independent scattering mechanisms. [S0163-1829(97)07011-2]
\end{abstract}

\section{INTRODUCTION}

In $\mathrm{GaAs} / \mathrm{Al}_{x} \mathrm{Ga}_{1-x} \mathrm{As}$ superlattices with narrow minibands, the Coulomb interaction contributes significantly to the localization of the $1 s$ heavy-hole exciton (HHX) states along the superlattice growth direction. ${ }^{1}$ At the same time, the state overlap between neighboring quantum wells of the superlattice remains considerable, and interwell Coulomb coupling results in a pronounced heavy-hole interwell excitonic (IWX) transition. ${ }^{2}$ This transition is resolved in the optical absorption spectra at photon energies a few meV above the HHX state. The linear optical properties near the band gap are thus dominated by two distinct excitonic resonances. One of them, the $1 s \mathrm{HHX}$, is spatially direct, and this state is confined mainly inside a single quantum well. The IWX is spatially indirect, and consists of an electron and a heavy hole in the nearest neighboring wells. Recently, the $1 \mathrm{~s}$ character of the IWX state was confirmed. ${ }^{3}$

Coherent interaction between spatially direct and indirect excitonic states is a topic, widely investigated in relation to Bloch oscillations. ${ }^{4}$ Transient four-wave mixing (TFWM) and nonlinear quantum beat spectroscopies have become standard tools for such studies. ${ }^{5}$ The narrow-miniband superlattices present a possibility to investigate such interactions in superlattices without the external electric field. Indeed, a pair of HHX and IWX states resembles partly the Stark ladder, which forms in superlattices, subject to an external electric field. Having in mind this analogy, one might expect that the quantum beats between the $\mathrm{HH}$ and IW excitons would indicate periodic oscillations in the spatial separation between the photoexcited electron and heavy hole along the superlattice direction. However, previously published experimental data on coherent exciton dynamics in narrowminiband superlattices ${ }^{6,7}$ have suggested, that the IW excitonic transition is not a discrete resonance, but has a continuumlike character. Due to this, no periodic beating with the $\mathrm{HH}$ exciton is observed in the TFWM experiment. Only under the influence of external factors, such as electric ${ }^{6}$ or magnetic ${ }^{7}$ fields, has the IWX acquired sufficient discreteness allowing for periodic beats with the HHX.

In this paper, we study excitonic dephasing and quantum beats in narrow-miniband superlattices using spectrally re- solved TFWM. Taking advantage of the spectral information, we confirm the discrete nature of the IWX in the investigated superlattice. We observe a resonant enhancement due to the IWX in the TFWM spectra, and a fast signal decay at this energy, indicating a large homogeneous broadening of the IWX resonance. Since the broadening is nearly independent of the excitation intensity, it is mostly due to scattering mechanisms such as phonon and defect scattering. Quantum beats with a period corresponding to the HHX-IWX energy difference are observed in the TFWM decay.

\section{SAMPLE AND EXPERIMENTAL TECHNIQUES}

The investigated superlattice, grown by molecular-beam epitaxy on a (100)-oriented semi-insulating GaAs substrate, consists of 20 periods of $\mathrm{GaAs} / \mathrm{Al}_{0.3} \mathrm{Ga}_{0.7} \mathrm{As}$. The thickness of the GaAs quantum wells is $L_{w}=80 \AA$, and the thickness of the $\mathrm{Al}_{x} \mathrm{Ga}_{1-x} \mathrm{As}$ barriers is $L_{b}=30 \AA$. To allow for linear and nonlinear optical measurements in transmission geometry, the sample was lifted off the GaAs substrate, by selective etching, and mounted on a sapphire disk. Photoluminescence (PL) was excited using the 632.8-nm line of a $\mathrm{HeNe}$ laser, and for transmission measurements, white light from a tungsten halogen lamp was used. The luminescence and transmission spectra were recorded by an optical multichannel analyzer (OMA) system. The inhomogeneous linewidth of the HHX line is $2.2 \mathrm{meV}$ (full width at half maximum) and shows a Stokes shift of the PL of $0.5 \mathrm{meV}$. All experiments were carried out at a temperature of $5 \mathrm{~K}$, which was maintained in a helium bath cryostat.

The TFWM experiments are performed using femtosecond pulses from a self-mode-locked Ti:sapphire laser with a $76-\mathrm{MHz}$ repetition rate. The duration of the transformlimited pulses was about $150 \mathrm{fs}$, and their central frequency was tuned to excite the HHX and IWX simultaneously. The two-beam self-diffraction geometry ${ }^{8}$ and time-integrated signal detection were used in the TFWM experiments. The signal was recorded in the nearly phase-matched direction $2 \mathbf{k}_{2}-\mathbf{k}_{1}$, where $\mathbf{k}_{1}$ and $\mathbf{k}_{2}$ are the wave vectors of the incident laser pulses, as a function of their mutual time delay $t_{12}$. For a three-level system, the decay of the TFWM signal 
intensity $I_{\mathrm{TFWM}}$, at the energy of one resonance, can be expressed as $^{9}$

$$
I_{\mathrm{TFWM}}\left(t_{12}\right) \propto \exp \left(-\frac{c t_{12}}{T_{2}}\right)\left[1+I_{m}\left(t_{12}\right) \cos \left(\frac{E_{1}-E_{2}}{\hbar} t_{12}\right)\right],
$$

where the constant $c$ is 2 (4) for homogeneously (inhomogeneously) broadened systems, and $T_{2}$ is a phenomenological dephasing time of the given resonance. The expression in the square brackets accounts for the quantum beats. $E_{1}$ and $E_{2}$ are the energies of the beating resonances, and $I_{m}\left(t_{12}\right)$ denotes the delay-dependent amplitude of the signal modulation, which is determined by the properties (oscillator strengths, dephasing times, inhomogeneous broadenings) of both resonances. The energy difference $E_{1}-E_{2}$ can be determined from the oscillation period of $I_{\mathrm{TFWM}}\left(t_{12}\right)$. To obtain the maximum available information in the TFWM experiment, ${ }^{9}$ the signal is dispersed in a spectrometer, and its spectrum is recorded using the OMA system as a slow detector.

\section{RESULTS AND DISCUSSION}

Before discussing the experimental data, it is useful to compare briefly the circumstances, which lead to the formation of the IWX states in superlattices with and without an applied external electric field.

A superlattice can be regarded as a multiple quantum well (MQW) structure, in which the individual quantum wells are allowed to interact via thin barriers. Without an applied electric field, the delocalization of the single-particle states due to the overlap with the neighboring wells competes with the Coulomb interaction, which correlate the positions of electrons and holes. In the present work, we are particularly interested in such correlation between the electrons and the heavy holes (hh). Denoting the respective miniband halfwidths as $\Delta E_{e}$ and $\Delta E_{\mathrm{hh}}$, the relative strength of both effects can be estimated from the comparison of the combined superlattice miniband halfwidth $\Delta E=\Delta E_{e}+\Delta E_{\mathrm{hh}}$ to the exciton binding energy $E_{x}^{b(\mathrm{QW})}$ in an isolated quantum well of the same thickness. Also, the distinction of broad and narrow miniband superlattices can be based on such a comparison. ${ }^{1}$

In superlattices with broad minibands, i.e., where $\Delta E>E_{x}^{b(\mathrm{QW})}$, effects due to Coulomb interaction are insignificant compared to those of the interwell coupling. In such structures, both electron and heavy-hole single-particle states are delocalized in the superlattice growth direction, and the Coulomb correlation between these states results in almost three-dimensional heavy-hole exciton states. Hence, the distinction between spatially direct and indirect transitions is not relevant. However, in an external electric field, the translational invariance of the superlattice is lifted, and the minibands split into series of discrete localized levels, referred to as the Stark ladder. ${ }^{10,11}$ The energy levels and optical transitions in the Stark ladder in the absence of the excitonic effects are sketched in Fig. 1(a) for a model superlattice with five quantum wells. One spatially direct and several indirect transitions are possible between the Stark-ladder levels, provided the localization is not complete. At moderate electric fields and low temperatures, a series of excitonic peaks can b)

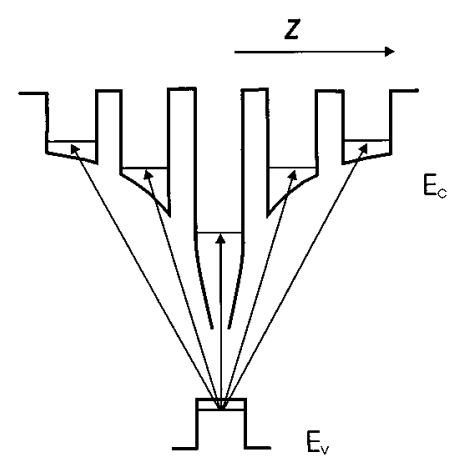

a)

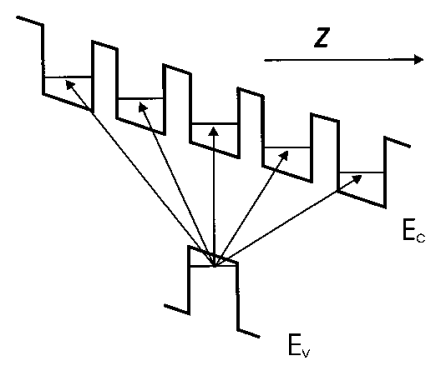

FIG. 1. Optical transitions in superlattices (a) in a broadminiband, electrically biased superlattice without the excitonic effects, and (b) in a narrow-miniband, electrically unbiased superlattices with excitonic effects. In both plots, the vertical transition is spatially direct, while the transitions, marked by the inclined arrows, are spatially indirect (interwell) transitions.

be seen in the absorption spectrum.

In narrow-miniband superlattices, where $\Delta E<E_{x}^{b(\mathrm{QW})}$ holds, the excitonic localization prevails over the delocalizing effect of the periodic superlattice potential. In this case, mainly the conduction-band single-particle states are delocalized, whereas the heavy-hole states due to their larger effective mass are confined in the quantum wells. The Coulomb interaction between photoexcited electrons and heavy holes effectively reduces the spread of the electron wave functions into the neighboring wells. ${ }^{1}$ Thus, even without an external electric field, the electrons are substantially localized to a single quantum well due to their correlation to the localized heavy holes, ${ }^{6}$ and their single-particle energy is at the middle of the miniband. Accordingly, the excitonic transition is found $E_{x}^{b(\mathrm{QW})}$ below the middle of the miniband, just as in an isolated quantum well. The basic optical properties of the narrow-miniband superlattices become obvious in a simple tight-binding model, ${ }^{6,7}$ which regards the Coulomb potential of the heavy hole being superimposed on the periodic superlattice potential. The above discussed localization is a result of locally broken translational symmetry of the superlattice. The energy levels and optical transitions according to this model are shown in Fig. 1(b).

For sufficiently strong interwell state overlap and Coulomb interaction, the IWX, composed of electrons and heavy holes in the nearest-neighboring wells become optically active. The optical transition to the IWX states is spatially indirect, and is shown in the same figure. The condition for the 


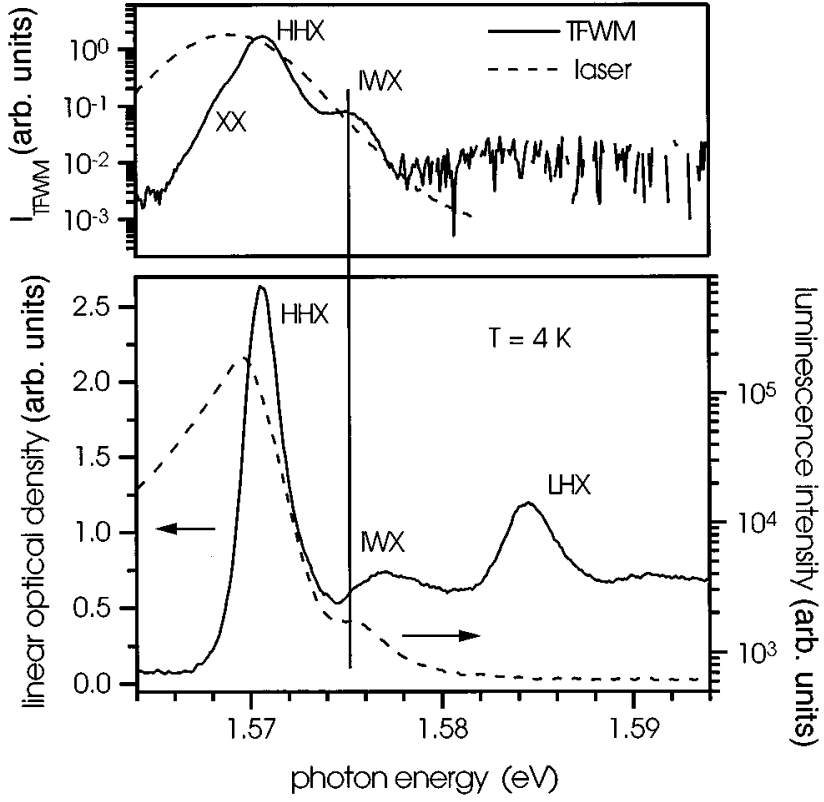

FIG. 2. Linear and nonlinear spectral characteristics of the narrow-miniband superlattice. The positions of the HHX, IWX, LHX, and biexciton (XX) are marked by labels.

formation of the IWX was outlined earlier to $b^{2}$

$$
\Delta E<E_{\mathrm{IW}} \sim E_{x}^{b(\mathrm{QW})} .
$$

Here, $E_{\mathrm{IW}}=e^{2} /\left[\epsilon_{0}\left(L_{w}+L_{b}\right)\right]$ is the interwell Coulomb interaction energy. The energy of the IWX state is lowered by the $e-h$ Coulomb interaction, but not as strongly as for the HHX state due to the finite $e-h$ separation in the growth direction. Interwell excitons are also formed by electrons and heavy holes separated by more than one superlattice period, if the Coulomb interaction energy between the particles satisfies the condition (2). However, the oscillator strength for these transitions will decrease rapidly with increasing interwell distance, thus making them very weak in optical spectra. ${ }^{6}$

\section{A. Quantum beats between HH and IW excitons}

For the superlattice parameters given in the previous section, the electron and heavy-hole miniband half-widths are $\Delta E_{e}=5.5 \mathrm{meV}$ and $\Delta E_{\mathrm{hh}}=0.4 \mathrm{meV}$, as obtained from $\mathbf{k} \cdot \mathbf{p}$ band-structure calculations. The HHX binding energy in 80$\AA$ A-wide noninteracting multiple quantum wells $E_{x}^{b(2 \mathrm{D})}$ was determined earlier ${ }^{12}$ to be $\approx 10 \mathrm{meV}$. These values show that the investigated sample falls into the category of narrowminiband superlattices. As expected, only the conductionband states are delocalized in the superlattice. The small width of the heavy-hole miniband indicates that the heavyhole states are essentially confined inside the wells. The interwell Coulomb interaction energy is $E_{\mathrm{IW}} \approx 10 \mathrm{meV}$ in this sample, hence the criterion (2) for the formation of IWX is fulfilled.

In Fig. 2, the spectra of the linear optical density, the cw PL, and the TFWM signal are shown. In the linear optical density, the HHX and light-hole excitons (LHX) are seen as peaks at 1.5706 and $1.5846 \mathrm{eV}$, respectively. The feature at $1.5768 \mathrm{eV}$, between the HHX and LHX resonances, is the IWX state, partially overlapping with the onset of the heavy-

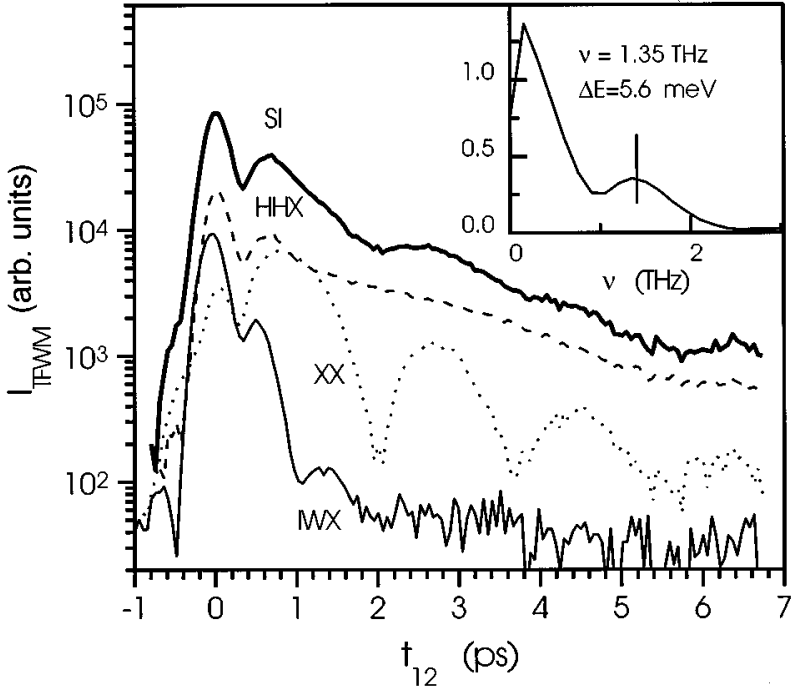

FIG. 3. Dynamics of the spectrally integrated and spectrally resolved TFWM signal. The spectrally integrated signal is labeled SI. The spectrally resolved signals at the energies of the HHX, biexciton (XX), and IWX are labeled. The $e-h$ pair density is $n_{e h}=4 \times 10^{9} \mathrm{~cm}^{-2}$. The inset shows the Fourier transform of the TFWM decay at the IWX energy with the peak at $1.35 \mathrm{THz}$.

hole continuum transitions. The IWX state is better resolved in the PL data, where it is seen as a shoulder at $1.5755 \mathrm{eV}$. The TFWM signal spectrum, recorded at $t_{12}=0.4 \mathrm{ps}$ and the spectrum of the laser pulse, used in the experiments, are shown in Fig. 2. The resonant enhancement due to the IWX state is clearly seen in the TFWM spectrum. From these data, a difference of about $5 \mathrm{meV}$ between the energies of the HHX and IWX states can be extracted.

Figure 3 shows the decay of the TFWM signal with delay time at several spectral positions. For comparison with previously published data, a spectrally integrated TFWM decay is also shown. The spectrally integrated signal exibits a peak around $t_{12} \approx 0 \mathrm{ps}$, a dip at $t_{12} \approx 0.35 \mathrm{ps}$, and subsequent weak oscillations with a period of about $1.7 \mathrm{ps}$, indicating quantum beats between levels, separated in energy by $\approx 2.4 \mathrm{meV}$. The linear spectra in Fig. 2 have no prominent spectral features separated by this energy.

In general, the initial part of the spectrally integrated decay is very similar to that found in $\mathrm{In}_{x} \mathrm{Ga}_{1-x} \mathrm{As} / \mathrm{GaAs}$ multiple quantum wells, where the HHX and the heavy-hole continuum transitions were excited simultaneously. ${ }^{13}$ The dip shortly after $t_{12}=0$, followed by partial recovery of the TFWM signal was regarded as a signature of quantum interference between the discrete resonance and the continuum. Such a feature was also observed in TFWM experiments on GaAs/ $/ \mathrm{Al}_{x} \mathrm{Ga}_{1-x} \mathrm{As}$ superlattices, ${ }^{7}$ and ascribed to the interference between the HHX resonance and continuumlike interwell transitions. It was shown in the same publication that in an external magnetic field, the IWX state displays features of a discrete resonance. An increase in the magnetic field results in a gradual transformation from the single dip to the periodic oscillations in the signal decay.

It was found previously that the HHX binding energy in the superlattice investigated here is about $7 \mathrm{meV} .{ }^{12}$ Hence, the onset of the HHX continuum transitions is just above the IWX resonance. In the TFWM measurements with subpico- 
second time resolution, it is not possible to excite selectively only the IWX exciton. Some continuum states are excited as well, and in principle they may affect our observations. However, the data presented below suggest that major features of the inital part of the decay are due to the quantum beats between two discrete resonances, namely, the HHX and IWX sates.

The decay of the spectrally resolved TFWM signal at several selected spectral positions explains the peculiarities observed in the spectrally integrated signal. The slow oscillations are strongly present about $2.5 \mathrm{meV}$ below the HHX transition energy, and they are ascribed to quantum beats between the HHX and biexcitons. ${ }^{14}$ The biexcitonic resonant enhancement can be seen as a low-energy shoulder in the TFWM signal spectrum in Fig. 2. The biexciton binding energy of $2.4 \mathrm{meV}$ is determined from the period of the beats. The biexcitonic resonance is inherently nonlinear and is therefore not seen in the linear spectra in Fig. 2, recorded at low excitation levels. The high biexciton binding energy of $2.4 \mathrm{meV}$ in the superlattice is essentially the same as was found in noninteracting multiple quantum wells with $L_{w}=80 \AA .{ }^{12}$ This is an indication that the heavy-hole biexcitons as well as the excitons are mainly localized inside the quantum well and are thus quasi-two-dimensional states.

The signal decay at the HHX energy, shown in Fig. 3, is nearly exponential for delays $t_{12}>1.5 \mathrm{ps}$. For shorter times, the decay is identical to that of the spectrally integrated signal. At the energy of the IWX resonant enhancement, the decay is much faster, and the signal exibits oscillations with a period of $0.73 \mathrm{ps}$. Accordingly, the Fourier transform of the signal decay at the IW exciton energy given in the inset shows a peak at a frequency of $1.355 \mathrm{THz}$, corresponding to $5.7-\mathrm{meV}$ energy splitting. This energy agrees with the difference between the HHX and IWX energies, estimated from Fig. 2, suggesting that the oscillations represent quantum beats between the two excitons. As can be seen from Fig. 3, the signal decay at the IWX energy is very fast, indicating large homogeneous broadening. Nevertheless, the oscillations at this energy indicate that the IW exciton state is a discrete resonance. The single dip in the initial part of the spectrally integrated and the HH excitonic TFWM signal can be qualitatively explained by the difference in the decay rates of the HHX and IWX resonances. The difference in the signal magnitude at the corresponding spectral positions increases rapidly with delay time, and only a single minimum is observed at the energy of the strong HHX excitonic resonance. The much weaker IWX exhibits two oscillations before reaching the noise level. However, by spectrally resolving the TFWM signal, we are able to separate this contribution.

\section{B. Intensity-dependent TFWM experiments}

The fast TFWM signal decay at the spectral position of the IW exciton points towards strong scattering of this state. In order to investigate its origin, we have performed TFWM experiments at different excitation intensities. The main idea of these experiments is to distinguish between the intrinsic and the density-induced scattering contributions. Also, spectrally resolved TFWM allows one to compare the IW exciton scattering to that of the $\mathrm{HH}$ exciton. The density dependence of the homogeneous linewidth $\gamma=1 / T_{2}$ is often expressed as ${ }^{15}$

$$
\gamma\left(n_{e h}\right)=\gamma(0)+A n_{\mathrm{eh}},
$$

where $n_{e h}$ is the $e$-h pair density, $\gamma(0)$ is the low-density intrinsic linewidth due to scattering by defects and phonons, and $A$ is a constant, characterizing the intensity-dependent scattering between various electronic states, populated by intense excitation.

The plots in Fig. 4 show the TFWM signal at different $e-h$ pair densities. The TFWM spectra for a fixed delay $t_{12}=0.4 \mathrm{ps}$ are shown in Fig. 4(a). An increase in the $e-h$ pair density results in a gradual increase in the homogeneous linewidth of the HHX peak. At the highest $e-h$ pair densities, when the homogeneous linewidth becomes comparable to the inhomogeneous linewidth $(\approx 2 \mathrm{meV})$, the broadening becomes apparent in the TFWM spectrum. The increase in the homogeneous linewidth of the HHX results in faster decay of the signal. This is illustrated in Fig. 4(b). The straight lines in the figure are fits to the exponential final parts of the signal decay, their slopes yield the signal decay rates $c \gamma$ [see Eq. (1)]. In Fig. 4(c), the same dependence is shown for the IWX. With increasing $e-h$ pair density, the homogeneous linewidths of both excitons increase, and as a result, the much weaker IWX resonance enhancement disappears gradually from the TFWM signal spectrum in Fig. 4(a). Correspondingly, the temporal oscillations in Figs. 4(a) and 4(b) become weaker.

The signal decay rate is shown in Fig. 5 as a function of the $e-h$ pair density for both types of excitons. It would be more instructive to determine this dependence for the homogeneous linewidth alone. However, at least in the case of the HHX, there is an apparent transition from predominantly inhomogeneous to homogeneous broadening with increase in the $e-h$ pair density. Hence, the constant $c$ in Eq. (1) undergoes a change from 4 to 2 . For this reason, we compare the decay rates $c \gamma$ of both resonances, and approximate them with a dependence of the form (3), as shown in Fig. 5.

The HH exciton is predominantly inhomogeneously broadened at low densities. The low-density homogeneous linewidth $\gamma_{\mathrm{HHX}}$ can be estimated from Fig. 5 to be $\approx 0.15 \mathrm{meV}$, which is a typical low-temperature value for the quasi-two dimensional HHX in narrow quantum wells. ${ }^{15}$

It is more difficult to determine the broadening of the IWX exciton due to the relative weakness of this resonance. Assuming homogeneous broadening $(c=2)$, one obtains the homogeneous linewidth $\gamma_{\mathrm{IWX}}$ of $1.8 \mathrm{meV}$, i.e., one order of magnitude larger than $\gamma_{\mathrm{HHX}}$. This linewidth corresponds approximately to the width of the IWX feature, observed in the luminescence and TFWM spectra (see Fig. 2). Therefore, the assumption about predominantly homogeneous character of the IWX seems to be reasonable.

It can be seen from Fig. 5, that fast dephasing and, correspondingly, large homogeneous broadening of the IWX resonance is intrinsic to the investigated structure. There is a big difference between the low-density homogeneous linewidths of the IWX and the HHX states. In contrast, the densitydependent scattering contributions are similar for both resonances. There might be several reasons for the observed 

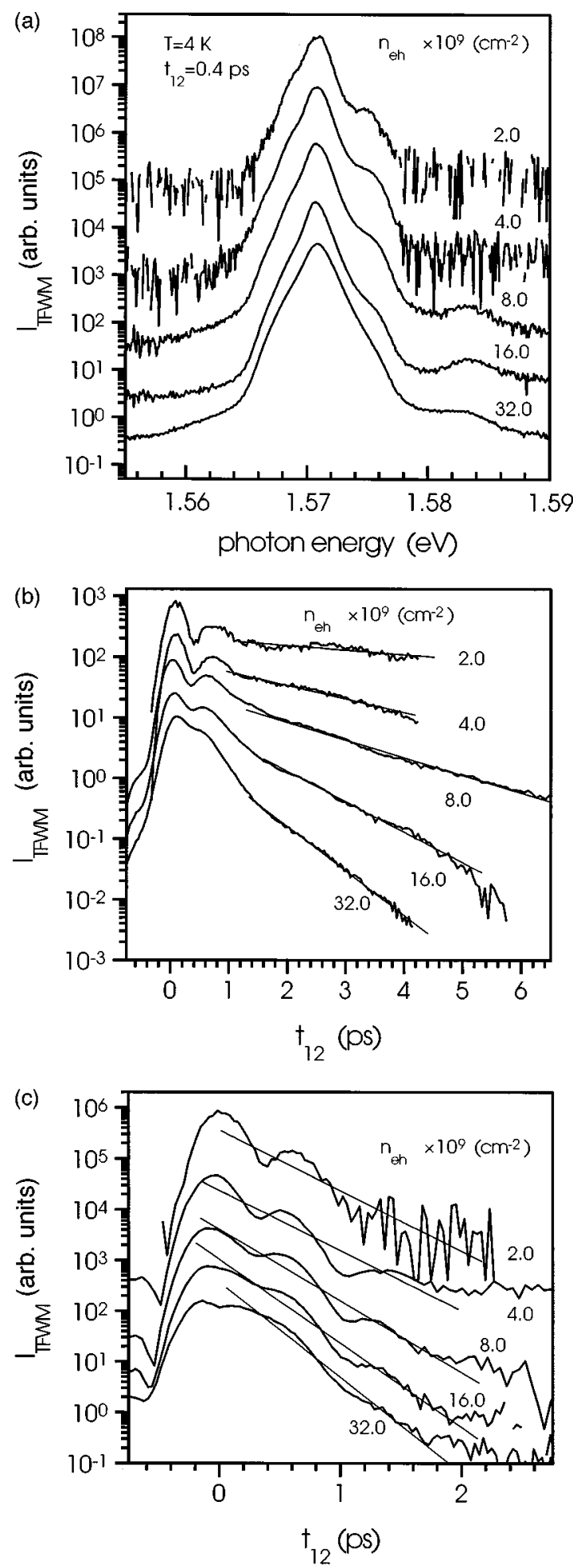

FIG. 4. Spectral and temporal shape of the TFWM signal at different excitation densities. The TFWM spectra at the delay $t_{12}=0.4 \mathrm{ps}(\mathrm{a})$, the signal decay at the HHX energy (b), the same at the IWX energy (c). The numbers above the curves indicate the $e-h$ pair density. The straight lines in plots (b) and (c) are exponential fits to the signal decay.

large intrinsic homogeneous linewidth of the IWX exciton. The IWX has a charge separation in the superlattice growth direction, and therefore should couple strongly to the LO phonons. Also, the exciton-polaron relaxation to the HHX

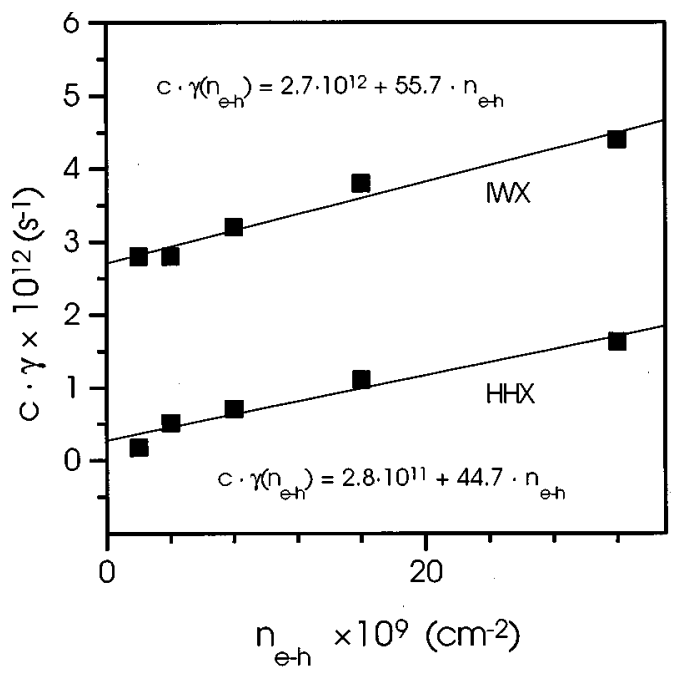

FIG. 5. TFWM signal decay rate at the IWX and the HHX resonances as a function of the $e-h$ pair density. The symbol size corresponds to the experimental uncertainty.

exciton state under accoustic phonon emission leads to a lifetime broadening of the IWX, especially because their inplane wave functions are similar. The low-density value of $c \gamma$ given in Fig. 5 infers subpicosecond lifetime of the IW exciton. This assumption is partially supported by the results of the picosecond time-resolved luminescence experiments, performed on the same sample. The IW exciton was excited resonantly, and the luminescence was recorded with a streak camera. We have observed a significant increase in the HHX luminescence, when the excitation was resonant with the IWX, but no luminescence was found at the IWX energy. This indicates fast IWX relaxation to the HHX, and the IWX lifetime due to the relaxation being shorter than the experimental time resolution of $20 \mathrm{ps}$.

Altogether, the data presented above support that the interwell exciton behaves as a discrete resonance. The observed beating between the IWX and the HHX has a peculiar character, because it involves spatially direct and indirect optical transitions, and resembles quantum beats between the Stark-ladder states in electrically biased, broad-miniband superlattices. It is well known that quantum beats in the Stark ladders are associated with Bloch oscillations in the external electric field. ${ }^{4}$ As can be seen from Fig. 1, the common feature between the Stark ladder and the excitonic localization is a broken translational invariance of the superlattice. In the former case, the external electric field acts as a global symmetry-breaking factor, which is also responsible for the spatial oscillations. The excitonic effects break the translational invariance locally, and in the vincinity of the photoexcited hole the electron state is strongly perturbed by the Coulomb field of the heavy hole. It might be expected that the beats between the $\mathrm{HH}$ and IW excitons, observed in the present studies, would correspond to the periodic spatial oscillations of the electrons in the local field of the heavy holes. However, by examining Fig. 1(b) one can see that even if the superlattice translational invariance is broken, the reflection symmetry is preserved, and a macroscopic oscillating dipole, characteristic for the Bloch oscillations, will not appear in this case. 


\section{CONCLUSIONS}

We have studied the coherent optical properties of a $\mathrm{GaAs} / \mathrm{Al}_{x} \mathrm{Ga}_{1-x} \mathrm{As}$ narrow-miniband superlattice by spectrally resolved transient four-wave mixing. These properties are strongly affected by the existence of the interwell heavyhole excitons. The IW exciton is revealed as a resonant enhancement of the TFWM signal. This TFWM signal has a fast decay, indicating predominant homogeneous broadening of the interwell exciton. The broadening is not related to the effects of high carrier density. We observe quantum beats between the $\mathrm{HH}$ and IW excitons in the TFWM signal, demonstrating the discrete nature of the IW exciton in the investigated structure. This finding is in contrast to the earlier results, ${ }^{7}$ which suggested that in the absence of an external field, interwell excitons contribute to the TFWM signal in a continuumlike fashion. The observed beating is between spatially direct and indirect optical transitions and can be regarded as an analog to the quantum beats between the Starkladder levels. However, the Bloch oscillations do not occur due to the reflection symmetry of the Coulomb potential.

The incoherent properties of the interwell exciton is currently under investigation using light-induced grating techniques. The aim of these studies is to investigate whether the tunneling of the interwell exciton into the direct exciton state is limiting the lifetime of the interwell exciton.

\section{ACKNOWLEDGMENTS}

This work was supported by the Danish Ministries of Industry and Research in the framework of CNAST. The sample used was grown by C.B. Sørensen, III-V Nanolab.
${ }^{1}$ A. Fox et al., Phys. Rev. B 46, 15365 (1992).

${ }^{2}$ D. Birkedal et al., Nuovo Cimento 17D, 1359 (1995).

${ }^{3}$ D. Birkedal et al., Phys. Rev. B 5410316 (1996).

${ }^{4}$ P. Leisching et al., Phys. Rev. B 50, 14389 (1994).

${ }^{5}$ K.-H. Pantke and J. M. Hvam, J. Mod. Phys. B 8, 73 (1994).

${ }^{6}$ G. Cohen, I. Bar-Joseph, and H. Shtrikman, Phys. Rev. B 50, 17316 (1994).

${ }^{7}$ G. Cohen, H. Shtrikman, and I. Bar-Joseph, Phys. Rev. B 52, 11642 (1995).

${ }^{8}$ L. Schultheis, M. D. Sturge, and J. Hegarty, Appl. Phys. Lett. 47, 995 (1985).
${ }^{9}$ J. Erland et al., Phys. Rev. B 50, 15047 (1994).

${ }^{10}$ E. Mendez, F. Aguilló-Rueda, and J. Hong, Phys. Rev. Lett. 60, 2426 (1988).

${ }^{11}$ P. Voisin et al., Phys. Rev. B 61, 1639 (1988).

${ }^{12}$ V. Mizeikis et al., Phys. Rev. B 55, 5284 (1997).

${ }^{13}$ J. Feldmann et al., Phys. Rev. Lett. 70, 3027 (1993).

${ }^{14}$ K.-H. Pantke et al., Phys. Rev. B 47, 2413 (1993).

${ }^{15} \mathrm{~J}$. Kuhl et al., in Coherent Optical Interactions in Semiconductors, edited by R. Phillips (Plenum, New York, 1994), pp. 1-31. 